\title{
La coexistence de procédures contentieuses en matière d'investissements étrangers
}

Fecha de recepción: 12 de noviembre de 2014

Fecha de aceptación: 4 de febrero de 2016

Doi: 10.12804/revistas.urosario.edu.co/acdi/a.5291

\section{Mathias Audit*}

Resume: Le développement considérable de l'arbitrage en matière d'investissements étrangers est l'une des évolutions les plus notables du droit international contemporain. Les Etats voient désormais régulièrement engager leur responsabilité devant des juridictions arbitrales lorsque des investisseurs estiment être victimes de leur comportement. Les modalités possibles de saisine de ces juridictions sont toutefois diverses; les instances peuvent être fondées sur une clause compromissoire ou sur une offre publique d'arbitrage d'un traité, multilatéral ou bilatéral, ou d'une loi nationale. Il en résulte que pour une même affaire ou pour des affaires proches, plusieurs tribunaux sont aujourd'hui fréquemment saisis. La présente contribution vise à présenter ces situations complexes, ainsi que les solutions qui pourraient ici être apportées.

Mots clefs: Arbitrage international, ALENA, autorite de chose jugée, CIRDI, CNUDCI, investissements étrangers, litispendance, Traité bilatéral de protection et de promotion des investissements (TBI).

* Professeur à l'Ecole de droit de la Sorbonne (Université Paris 1). Email: mathias.audit@ wanadoo.fr

Para citar este artículo: Audit, M., «La coexistence de procédures contentieuses en matière d'investissements étrangers", Anuario Colombiano de Derecho Internacional (ACDI), 2017, 10, pp. 333-370. Doi: 10.12804/revistas. urosario.edu.co/acdi/a.5291 
Abstract: Massive development of arbitration in foreign investment is one of the most significant developments in contemporary international law. Foreign investors are now regularly suing States by arbitration tribunals. However, there are different ways of filing arbitration cases in this respect. They can be grounded on arbitration clauses or on the basis of multilateral or bilateral treaties, even on the respondent state own domestic law. As a result, for same or near cases, several tribunals might be involved. This paper aims to present these complex situations and the solutions that could be proposed in this respect.

Key words: International arbitration -NAFTA — res judicata, ICSID, Uncitral, foreign investment, lis pendens, Bilateral Investment Treaty (ВIT).

La coexistencia de procedimientos contenciosos en materia de inversión extranjera

Resumen: el gran desarrollo del arbitraje en materia de inversión extranjera es uno de los aportes más notables del derecho internacional contemporáneo. Los Estados ven regularmente cómo se determina su responsabilidad ante las jurisdicciones arbitrales cuando los inversores estiman haber sido víctimas de su comportamiento, sin embargo, las posibles modalidades de tomar estas jurisdicciones son diversas. Las instancias pueden estar fundadas sobre una cláusula compromisoria o sobre una oferta pública de arbitraje de un tratado multilateral o bilateral, o aun con una ley nacional. Puede resultar que para un mismo caso o para casos similares varios tribunales puedan tener jurisdicción para conocer de ellos. Esta contribución busca presentar estas situaciones complejas, así como aportar posibles soluciones.

Palabras clave: arbitraje internacional (NAFTA), autoridad de la cosa juzgada, Centro Internacional de Arreglo de Diferencias Relativas a Inversiones (CIADI), Comisión de las Naciones Unidas para el Derecho Mercantil Internacional (CNUDMI), inversión extranjera, litispendencia, Tratado Bilateral de Inversión (TBI). 
A coexistência de procedimentos contenciosos em matéria de investimento estrangeiro

Resumo: O grande desenvolvimento da arbitragem de investimento estrangeiro é um dos aportes mais notáveis do Direito Internacional Contemporâneo. Os estados veem regularmente como se determina a sua responsabilidade ante as jurisdições arbitrais quando os inversores consideram ter sido vítimas do seu comportamento, no entanto, as possíveis modalidades de tomas estas jurisdições são diversas. As instâncias podem estar fundadas sobre uma cláusula compromissória ou sobre uma oferta pública de arbitragem de um tratado multilateral ou bilateral ou ainda com uma lei nacional. Pode resultar que para um mesmo caso ou para casos similares vários tribunais possam ter jurisdição para conhecer deles. Esta contribuição busca apresentar estas situações complexas, assim como aportar possíveis soluções.

Palavras-chave: Arbitragem internacional (NAFTA), autoridade da coisa julgada, Centro Internacional para a Arbitragem de Disputas sobre Investimentos (CIADI), Comissão das Nações Unidas para o Direito Mercantil Internacional (CNUDMI), investimentos estrangeiros, litispendência, Tratado Bilateral de Investimento (TBI).

I. Les options contentieuses offertes aux investisseurs

A. Option contentieuse entre une offre générale d'arbitrage et la saisine d'une juridiction interne

1. La saisine possible des juridictions internes

2. Clause d'electa una via

B. Option contentieuse entre une offre générale d'arbitrage et une clause attributive de juridiction ou une clause compromissoire

C. Option contentieuse issue d'un même traité ou d'une même loi de protection des investissements étrangers

1. Clause d'arbitrage multiple

2. Recours au traitement de la nation la plus favorisée à des fins procédurales

II. La concurrence des procedures contentieuses

A. Consolidation des procédures contentieuses

1. Consolidation institutionnellement organisée 
2. Consolidation à l'initiative des parties ou du tribunal arbitral B. Concurrence avec une procédure en cours

1. Litispendance (Lis pendens)

2. Débat sur l'avènement de la connexité

C. Concurrence avec une décision antérieure

1. Effet négatif de chose jugée

2. Effet positif de chose jugée

En matière de règlement des différends liés aux investissements étrangers, la coexistence de procédures contentieuses ou l'opportunité d'une telle coexistence sont des situations qui, dans la pratique, s'avèrent finalement très fréquentes. D'une manière très générale, il est vrai que tous les litiges transnationaux, quel que soit leur objet, donnent bien souvent prise à des configurations de ce type. Le forum shopping y est commun et la saisine concomitante de plusieurs juridictions nationales ou arbitrales, habituelle. Mais indéniablement, toutefois, le contentieux en matière de protection des investissements étrangers présente à cet égard une singularité supplémentaire. Les opportunités qu'il offre en matière d'instances concomitantes ou successives y sont, à l'évidence, plus importantes encore qu'en d'autres matières contentieuses.

Ce constat s'explique aisément. En se limitant à la seule saisine de tribunaux arbitraux, il existe en matière de protection des investissements étrangers trois fondements possibles à leur compétence: un contrat d'État, un traité (TBI ou AMI) ou une loi de l'État d'accueil. ${ }^{1}$ Dès lors, à l'occasion d'une même opération d'investissement, deux ou même trois de ces différentes procédures arbitrales sont susceptibles d'être engagées. Si l'investissement a fait l'objet d'un contrat avec l'État d'accueil ou l'une de ses émanations et qu'il entre par ailleurs dans le champ d'application d'un TBI, d'un AMI ou d'une loi nationale, toutes les procédures arbitrales prévues par ces différents instruments peuvent être envisagées et elles sont en principe susceptibles d'être concomitamment introduites.

Par ailleurs, des recours devant des tribunaux internes sont également possibles. Par application d'une clause attributive de juridiction, le litige contractuel peut en particulier être porté devant une juridiction étatique. On peut même envisager qu'une action non contractuelle, en responsabilité

1 Sur ces différents chefs de compétence arbitrale possibles et les régimes distincts qui en découlent, v. not. M. Audit, «Droit des investissements internationaux. Contentieux arbitral international. Droit commun», JCL Droit international, fasc. 572-65, $\mathrm{n}^{\circ} 7$ et s. 
extracontractuelle ou de nature pénale ou administrative par exemple, ait été introduite à l'encontre de l'investisseur étranger devant les tribunaux de l'État d'accueil. À cette présentation des recours possibles, il peut même être envisagé que, pour la même affaire, une instance interétatique ait été introduite sur le fondement de la protection diplomatique. Dans l'affaire Lucchetti, par exemple, le Pérou a diligenté une procédure d'arbitrage — interétatique donc — à l'encontre du Chili, concomitamment à la procédure CIRDI en cours entre l'investisseur chilien et le premier État. ${ }^{2}$

Il existe donc en la matière une possible démultiplication des opportunités contentieuses. S'il est une affaire qui est susceptible de l'illustrer, c'est très certainement le litige qui a opposé la société italienne Saipem à l'État du Bangladesh. ${ }^{3}$ Dans un premier temps, l'investisseur avait ici commencé par introduire une requête en arbitrage commercial devant la CCI à l'encontre de son contractant direct, c'est-à-dire la Bangladesh Oil Gas and Mineral Corporation (Petrobangla) qui est une émanation de l'État bengali créée par voie législative.

Parallèlement à cette première procédure arbitrale, ladite émanation a successivement saisi plusieurs juridictions bengalies afin que soit enjoint tant à la société Saipem qu'au tribunal ccı lui-même d'interrompre la procédure arbitrale. Celui-ci n'a toutefois pas obtempéré, finissant par prononcer une sentence sur le fond emportant condamnation de la société Petrobangla. Saisie par cette dernière société, la High Court Division of the Supreme Court du Bangladesh a refusé de reconnaître à la décision du tribunal CCI la valeur d'une sentence arbitrale du commerce international. Par ailleurs, et concomitamment, différentes instances ont été introduites devant les juridictions bengalies par un sous-traitant de Saipem, tandis que cette dernière société saisissait de son côté les juridictions milanaises afin de prévenir l'appel en garantie bancaire que n'allait pas manquer de mettre en œuvre la partie bengalaise.

Or, à ces différentes procédures, est venue s'en adjoindre une autre puisque l'investisseur a fini par introduire une nouvelle requête arbitrale devant le CIRDI, mais sur le fondement cette fois-ci du traité bilatéral de protection des investissements conclu entre l'Italie et le Bangladesh. Dans

\footnotetext{
2 W. Ben Hamida, «L'arbitrage État-investisseur face à un désordre procédural: la concurrence des procédures et les conflits de juridictions», AFDI 2005, pp. 564-602, spéc. p. 576.

3 Saipem S.p.A. c. Bangladesh, aff. $\mathrm{n}^{\circ}$ ARB/05/7, SC du 21 mars 2007, ICSID Rev. 2007, p. 100; S. du 30 juin 2009.
} 
le cadre de celle-ci, il a alors principalement demandé au tribunal CIRDI de juger que l'interruption de la procédure cci par les juridictions bengalies soit considérée comme une expropriation au sens du TBI!

La nature de cette dernière demande met en exergue la singularité de cette affaire, qui l'éloigne finalement du sujet ici traité. Les procédures alternatives à celle entreprise sous l'égide du CIRDI n'y sont envisagées qu'au titre d'éléments factuels afin de déterminer si le TBI a été ou non violé. À aucun moment, le tribunal CIRDI ne s'est posé la question de l'incidence - en droit - sur sa propre procédure de la solution donnée dans les autres contentieux. Mais il n'en reste pas moins que cette affaire illustre à merveille l'incroyable enchevêtrement possible de procédures contentieuses pour une même opération d'investissement. Y sont réunis tous les ingrédients propres à la question des procédures parallèles dans l'arbitrage en matière d'investissements étrangers. Pour un même litige, ont été saisis un tribunal arbitral de la CCI, des juridictions internes, ressortissant tant à l'État hôte qu'à l'État d'accueil, et un tribunal arbitral statuant sous l'égide du CIRDI.

Or, il est évident que ce type de situation ne favorise pas le règlement global des contentieux nés d'opérations d'investissement. Bien au contraire, il en perturbe fortement la résolution définitive. L'objet de la présente étude est donc, à terme, de présenter les différents outils procéduraux susceptibles d'être mis en œuvre afin qu'une solution univoque à ce type de litiges puisse être obtenue. Mais, à cette fin, il importe de présenter les différentes options contentieuses qui sont offertes aux investisseurs (I). On constatera alors qu'elles sont nombreuses, ce qui explique l'existence fréquente d'une concurrence des procédures contentieuses (II).

\section{Les options contentieuses offertes aux investisseurs}

En présence d'un litige né d'une opération d'investissement, l'État ou son émanation dispose d'un choix procédural restreint. S'il existe un contrat conclu avec l'investisseur, le juge de celui-ci, fut-il étatique ou arbitral, peut être saisi. ${ }^{4}$ En l'absence de relations contractuelles directes entre l'État ou

\footnotetext{
4 En pratique, les affaires CIRDI où un État ou une entité étatique apparaissent en qualité de demandeurs sont toutefois très rares: Gouvernement de la Province du Kalmantan de l'Est c. PT Kalim Prima Coal, aff. n ARB/07/3; Gabon c. Sté Serete SA, aff. nº ARB/76/1; Tanzania Electric Supply Company Limited c. Independent Power Tanzania Ltd., aff. $\mathrm{n}^{\circ} \mathrm{ARB} / 98 / 8$, S. du 12 juillet 2001 .
} 
l'une de ses entités publiques et l'investisseur, les possibilités d'action se réduisent finalement à la saisine des juridictions étatiques, et ce sont généralement ses propres tribunaux qui sont privilégiés par la partie étatique. En revanche, la saisine d'un tribunal arbitral sur le fondement d'un TBI ou d'un AMI ne peut s'opérer à l'initiative de l'État d'accueil, même si celui-ci estime avoir subi un préjudice en lien avec l'opération d'investissement. Seuls les investisseurs étrangers peuvent en effet se prévaloir du mécanisme d'arbitrage prévu par ce type de traités.

D’une manière plus générale et dans la grande majorité des hypothèses, ce sont du reste ces investisseurs étrangers qui disposent du choix procédural le plus vaste. Cette situation est un atout, l'investisseur pouvant ainsi identifier la procédure a priori la plus favorable à la préservation de ses intérêts. Ce choix doit toutefois être opéré avec une grande circonspection, car bien souvent il présente un caractère définitif avec pour effet de lui fermer certaines alternatives procédurales.

On étudiera donc successivement les différentes options contentieuses offertes à l'investisseur. Il peut avoir à choisir entre une offre générale d'arbitrage et la saisine des juridictions de l'État d'accueil (A) ou entre une offre du même type et une clause de compétence (B). Enfin, même sur le fondement d'un seul et même traité ou d'une seule et même loi de l'État d'accueil, l'investisseur est tout de même fréquemment confronté à un choix contentieux $(\mathrm{C})$.

\section{A. Option contentieuse entre une offre générale d'arbitrage et la saisine d'une juridiction interne}

Les juridictions étatiques peuvent donc connaittre des différends relatifs aux investissements internationaux. Toutefois, d'un point de vue pratique, la saisine d'un juge national ne constitue pas nécessairement une alternative efficace pour régler ce type de contentieux. En outre, et d'un point de vue théorique cette fois-ci, lorsque de tels litiges sont portés devant des juridictions internes, ils se détachent du droit des investissements internationaux, leur problématique se dénouant alors plus volontiers sous l'égide des méthodes du droit international privé, ou même du droit public interne de la juridiction saisie. Il n'en reste pas moins que la saisine par l'investisseur des juridictions internes est toujours possible (1). Toutefois, un tel choix doit être opéré avec circonspection, car il est susceptible d'entrainer la mise en œuvre d'une clause d'electa una via (2). 


\section{La saisine possible des juridictions internes}

Lorsqu'une opération d'investissement étranger est affectée par le comportement de l'État d'accueil, l'investisseur a bien évidemment toujours la possibilité de saisir les juridictions nationales de cet État. Il peut par exemple contester la mesure d'expropriation ou de nationalisation affectant son investissement ou l'absence d'indemnisation qui s'en est suivie; il peut également déférer devant le juge local le refus ou le retrait d'une autorisation administrative d'exploitation indispensable au fonctionnement de son usine; plus généralement, il pourrait même tenter d'engager la responsabilité de l'État d'accueil devant ce même juge du fait de son activité législative ou administrative. Bref, il existe toutes sortes de contentieux que l'investisseur étranger est susceptible d'engager devant les tribunaux de l'État d'accueil de son investissement.

Mais, à tort ou à raison, l'investisseur se méfie généralement des juridictions locales. Il n'y aura recours qu'en l'absence d'alternative jugée, à ses yeux, satisfaisante. ${ }^{5}$ Certes, l'investisseur pourrait être tenté de saisir une juridiction interne, mais étrangère à l'État d'accueil, les tribunaux de son État d'origine notamment. La difficulté est alors qu'ainsi prises en tant que défenderesses à l'action introduite devant un juge qui leur est étranger, les autorités de l'État d'accueil seront en mesure de se prévaloir de l'immunité de juridiction. En outre, dans certains systèmes juridictionnels, la doctrine de l'Act of State est également de nature à faire échec à la procédure contentieuse. Ainsi, dans une décision restée célèbre, la Cour suprême des États-Unis a, sur le fondement de cette doctrine, refusé d'examiner la validité au regard du droit international d'une mesure d'expropriation adoptée par un gouvernement étranger pour des biens situés sur le territoire de celui-ci. ${ }^{6}$ En outre, même si ces différents écueils pouvaient être levés, il est douteux qu'une décision adoptée par une juridiction étatique étrangère, dans un litige relatif à une opération d'investissement dans un État donné, puisse être reconnue et rendue efficace sur le territoire de

\footnotetext{
Pour un exemple: High Court (Namibie), 6 mars 2008, Kessl et al. c. Minister of Lands and Resettlement, RD aff. int. 2008, p. 708, obs. F. Horchani, W. Ben Hamida et E. Cabrol.

6 Banco National de Cuba v. Sabbatino, 376 U.S. 398 (1964). Sur cette question, v. G. Sperduti, «Des actions judiciaires intentées dans un État du chef de nationalisations et d'expropriations opérées dans un autre État», in Mélanges Ch. Rousseau, Paris, Pedone, 1974, pp. 249-266.
} 
celui-ci. Le succès de la procédure de reconnaissance ou d'exequatur est très loin d'être assuré.

En matière de protection des investissements par un juge interne, seuls les tribunaux de l'État d'accueil paraissent constituer le for adéquat pour connaitre des griefs de l'investisseur. Mais la difficulté est alors que ces tribunaux ne sont pas toujours jugés comme très fiables par l'investisseur. Ceci explique qu'une alternative ait été recherchée en ayant recours à l'arbitrage et, à cet égard, on peut affirmer sans conteste que l'ensemble du droit des investissements internationaux a été édifié dans l'objectif d'offrir à l'investisseur étranger une alternative tant à la saisine du juge local qu'à l'application de la loi de celui-ci. ${ }^{7}$

Or, comme on le sait, ${ }^{8}$ depuis l'essor du mécanisme du consentement dissocié, les investisseurs étrangers ont la possibilité de se prévaloir des offres générales d'arbitrage posées par traité ou à la faveur d'une loi nationale. Aux termes de celles-ci, les États d'accueil s'engagent par avance à accepter la procédure arbitrale qui serait engagée à leur encontre par un investisseur s'estimant lésé. Lorsqu'elle existe - car encore faut-il qu'une telle offre ait été posée par une loi de l'État d'accueil ou par un traité ratifié par celui-ci et par l'État d'origine de l'investisseur -, elle constitue effectivement une alternative très intéressante pour l'investisseur à la saisine du juge local.

Mais il est possible que celui-ci ait, dans un premier temps, néanmoins tenté sa chance devant les juridictions locales avant de prendre finalement la décision d'avoir recours à l'arbitrage prévu par un traité ou une loi. Dans cette hypothèse, l'existence d'une clause d'electa una via pourrait entraîner le rejet de sa requête arbitrale.

\section{Clause d'electa una via}

De nombreux traités adoptés en matière de protection des investissements étrangers prévoient une clause electa una via également dénommée fork in the road dans la terminologie anglophone. Ce type de disposition a pour effet d'imposer au demandeur de choisir entre le recours à l'arbitrage et une procédure devant les juridictions de l'État d'accueil de l'investissement.

\footnotetext{
7 R. Dolzer and Ch. Schreuer, Principles of International Investment Law, Oxford University Press, Oxford, 2008, p. 214.

8 V. supra, la contribution de P. Mayer, «Le Consentement à l’arbitrage».
} 
Plus encore, lorsque l'une des branches de l'alternative a été retenue, elle emporte renonciation à l'autre.

En pratique, ce type de clause est assez fréquent, notamment dans les TBI. L'article 6-3, a, du TBI conclu entre la France et l'Arabie saoudite, le 26 juin 2002, prévoit ainsi la disposition suivante: «Si le différend est soumis au tribunal compétent de la Partie contractante, $[. .$.$] l'investisseur$ s'interdit de recourir simultanément à l'arbitrage international et la sentence du tribunal est exécutoire et non susceptible de faire l'objet d'appels ou de recours autres que ceux prévus par la législation de la Partie contractante». ${ }^{9}$ Autrement dit, en application de l'article 6 de ce TBI, l'investisseur peut décider soit de porter son litige devant les tribunaux internes de l'État sur le territoire duquel l'investissement a été réalisé, soit d'avoir recours à l'arbitrage, en l'espèce du CIRDI. Mais dans l'hypothèse où il privilégierait la première de ces deux options, il est alors expressément indiqué que la voie de l'arbitrage sur le fondement du TBI lui est alors définitivement fermée.

Toutefois, en pratique, l'effet de ce type de clause d'electa una via n'est pas aussi important que celui que l'on aurait tendance à lui attacher de prime abord. Pour qu'elle puisse être invoquée et conduire le tribunal arbitral à se déclarer incompétent sur son fondement, encore faut-il en effet que celui-ci ait été saisi du même «différend» que celui dont ont préalablement eu à connaître les juridictions de l'État d'accueil à l'initiative de l'investisseur.

Tout le débat quant à la portée exacte de ce type de clause porte sur cette identité de différend entre la procédure introduite devant une juridiction interne et celle qui est entreprise devant le tribunal arbitral prévu par le traité prévoyant ladite clause. En principe, cela suppose une «identité de parties, d'objet et de cause dans les procès interne et international». ${ }^{10} \mathrm{Or}$, à retenir cette conception stricte de l'identité de différend, la similitude de cause entre les deux procédures fera défaut dans la très grande majorité des hypothèses, pour ne pas dire systématiquement. Devant le juge national, c'est une violation du droit interne qui est en effet invoquée, tandis que c'est une violation du traité qui est soulevée devant le tribunal arbitral. Ce constat n'a pas échappé à la jurisprudence arbitrale. ${ }^{11}$

9 D. publ. n ${ }^{\circ}$ 2004-207 du 8 mars 2004, JORF 10 mars 2004, p. 4686.

10 C. Santulli, Droit du contentieux international, Domat droit public, Montchrestien, Paris, $2005, n^{\circ} 156$.

11 Enron Corporation and Ponderosa Assets c. Argentine, aff. n ARB/01/3, SC du 14 janv. 2004, spéc. \ 97. 
Sur le fondement de l'absence d'identité de cause, c'est ainsi qu'une clause d'electa una via sera généralement considérée comme inopérante en présence d'une action fondée sur un contrat (contract claim) introduite devant les tribunaux internes. Le tribunal arbitral estimera en effet que celle-ci est distincte de la requête fondée sur une violation du traité (treaty claim) dont il est saisi. Dans l'affaire CMS c. Argentine, les arbitres l'ont expressément relevé: "As contractual claims are different from treaty claims, even if there had been or there currently was a recourse to the local courts for breach of contract, this would not have prevented submission of the treaty claims to arbitration $\gg .{ }^{12}$

Il faut bien reconnaitre que cette interprétation très restrictive de la clause d'electa una via revient de fait à la priver de tout effet. En pratique, l'instance ouverte devant le juge interne ne vise jamais à apprécier le comportement de l'État d'accueil au regard des dispositions du traité de protection des investissements. Dès lors, l'identité de cause ne peut pas se vérifier. Ceci explique que certains tribunaux arbitraux aient retenu une interprétation plus libérale de la clause d'electa una via, tendant à s'écarter d'une conception trop stricte de l'identité de différend ou, à tout le moins, de cause. Telle a été notamment la position d'un comité ad hoc du CIRDI dans l'affaire Compañia de Aguas del Aconquija and Vivendi Universal S.A. c. Argentine. En s'appuyant sur les termes mêmes de la clause electa una via du TBI fondant en l'espèce la compétence du tribunal arbitral, il a considéré que ne limite pas son champ d'application aux seules violations du TBI luimême, mais à tout différend relatif à un investissement opéré sous l'égide $\mathrm{du}$ TBI: «It is sufficient that the dispute relate to an investment made under the BIT». ${ }^{13}$

\section{B. Option contentieuse entre une offre générale d'arbitrage et une clause attributive de juridiction ou une clause compromissoire}

Tous les investisseurs étrangers, loin s'en faut, ne concluent pas de contrat avec l'État d'accueil ou l'une de ses émanations. Plus encore, même

\footnotetext{
12 CMS Gas Transmission Company c. Argentine, aff. n ARB/01/8, SC du 13 juill. 2003, spéc. \80, ICSID Rep. 2003, p. 492, JDI 2004, p. 236, obs. E. Gaillard. V. ég. inter alia: Lauder c. Rép. tchèque, S. ad hoc du 3 sept. 2001, SS 161 et 162; Azurix Corp.c. Argentine, aff. n ARB/01/12, SC du 8 déc. 2003, spéc. \89, JDI 2004, p. 275, obs. E. Gaillard; Occidental Exploration and Production company c. Ecuator, S. LCIA du 1er juill. 2004, spéc. \52.

13 Compañia de Aguas del Aconquija and Vivendi Universal S. A. c. Argentina, aff. n ARB/97/3, DCAH du 3 juill. 2002, J 55, ICSID Rev. 2004, p. 89, JDI 2003, p. 195, obs. E. Gaillard.
} 
lorsqu'un tel contrat existe, n’y a pas nécessairement été stipulée une clause attributive de juridiction ou une clause compromissoire en cas de litige. Mais lorsque tel est le cas, la question se pose alors de l'articulation de ces clauses de compétence avec une offre générale d'arbitrage dont bénéficierait par ailleurs l'investisseur. Plus exactement, on peut se demander si l'existence d'une telle clause de compétence emporte la perte du bénéfice de ladite offre.

Il faut dire qu'en pratique, la présence de clauses compromissoires ou de clauses attributives de juridiction dans des contrats soumis à l'appréciation de tribunaux arbitraux, saisis sur le fondement de traités de protection des investissements, est assez fréquente. Plus encore, dans un tout premier temps, certains tribunaux arbitraux n’ont pas été insensibles aux arguments développés à l'appui de cet effet renonciateur de la clause de compétence, sans finir toutefois par l'admettre expressément.

La question s'est notamment dans les affaires Lanco c. Argentine ${ }^{14}$ et Salini c. Maroc, ${ }^{15}$ où des tribunaux arbitraux saisis sur le fondement de TBI ont été confrontés à la stipulation de clauses attributives de juridiction dans les contrats constitutifs des opérations d'investissement litigieuses. Dans les deux cas, il s'agissait de contrats de nature administrative, relatifs à la concession d'un terminal portuaire à Buenos Aires dans la première affaire et à la construction d'une autoroute au Maroc dans la seconde, et ceux-ci soumettaient expressément tout litige né de leurs dispositions aux tribunaux administratifs de l'État en cause. On était donc en présence de clauses attributives de juridiction dans ces deux affaires, quoique d'une nature assez particulière puisqu'elles désignaient un juge administratif, et non judiciaire.

Quoi qu'il en soit, en particulier dans l'affaire Lanco, le tribunal arbitral a expressément constaté qu'un tel choix en faveur du juge administratif local ne constitue pas, à proprement parler, la manifestation d'une autonomie de la volonté propre aux parties contractantes. En effet, comme dans la plupart des systèmes juridiques disposant d'un ordre juridictionnel administratif, sa compétence est en réalité impérative en présence d’un

\footnotetext{
14 Lanco International, Inc. c. République Argentine, aff. n ARB/97/6, SC du 8 décembre 1998, ILM 2001, vol. 40, pp. 457-473.

15 Salini Construttori S.p.A. et Italstrade S.p.A. c. Royaume du Maroc, aff. ${ }^{\circ}$ ARB/00/4, SC du 23 juillet 2001, JDI 2002, pp. 196-209. Dans cette dernière affaire, le contrat comportant la clause attributive de juridiction avait été signé par une entité publique indépendante de l'État.
} 
contrat relevant du droit administratif. ${ }^{16}$ Dans ces conditions, la clause attributive de juridiction n'est pas, pour le tribunal arbitral, significative d'une quelconque option exercée par l'investisseur. De tels motifs donnent à penser qu'en présence d'une véritable manifestation de volonté en faveur du juge local, le tribunal eut, dans l'affaire Lanco de 1998, peut-être jugé différemment.

Mais, de toute façon, il est à peu près certain qu'eût-il été expressément entériné à l'époque, l'effet renonciateur de la clause de compétence doit de toute façon être envisagé différemment aujourd'hui. En effet, dans l'histoire finalement très récente de l'arbitrage en matière d'investissements étrangers fondé sur le principe du consentement dissocié, a entre-temps émergé la question de la distinction entre contract claims et treaty claims. Or, même si l'on sait que celle-ci fait l'objet d'un vif débat doctrinal, ${ }^{17}$ la jurisprudence arbitrale se montre en revanche plutôt univoque sur cette question. Elle tend à considérer que seules les demandes fondées sur la violation de dispositions substantielles du traité — treaty claims — sur le fondement duquel un tribunal arbitral a été saisi peuvent lui être adressées, c'est-àdire relèvent de sa compétence ratione materia. En revanche, les demandes de nature strictement contractuelle, c'est-à-dire tirées d'une violation du contrat unissant l'investisseur à l'État ou l'une de ses émanations et entrant en contradiction avec le droit qui lui est applicable — contract claims —, ne relèvent pas de la compétence d'un tribunal saisi sur le fondement d'un traité de protection des investissements. ${ }^{18}$

16 «[...] the Parties could not have selected the jurisdiction of the Federal Contentious-Administrative Tribunals of the City of Buenos Aires because it would hardly be possible to select the jurisdiction of courts whose own jurisdiction are, by law, not subject to agreement or waiver, whether territorially, objectively, or functionally. As contentious-administrative jurisdiction cannot be selected or waived, submission to the contentious-administrative tribunals cannot be understood as a previously agreed dispute-settlement procedure». Lanco International, SC préc. du 8 décembre 1998, spéc. \26, p. 466.

17 V. not. J. Crawford, «Treaty and Contract in Investment arbitration», Arb. Int. 2008, p. 351; I. Fadlallah, «La distinction Treaty claims - Contract claims et la compétence du CIRDI: faisons-nous fausse route ?», in Le contentieux arbitral transnational relatif à l'investissement, Ch. Leben (dir.), Paris, LGDJ/Anthemis, 2006, pp. 205-216; S. Lemaire, «Treaty claims et Contract claims: la compétence du CIRDI à l’épreuve de la dualité de l'État», Rev. arb. 2006, pp. 353-400; J.-P. Loncle, «L'option d'arbitrage des traités de protection des investissements: Treaty claims vs. Contract claims», RD aff. int. 2005, pp. 3-12; P. Mayer, "Contract claims et clauses juridictionnelles des traités relatifs à la protection des investissements », JDI 2009, pp. 71-96.

18 En ce sens, Bayindir Insaat Turizm Ticaret Ve Sanayi A.S. c. Pakistan, aff. $\mathrm{n}^{\circ}$ ARB/03/29, 
En l'état de cette jurisprudence arbitrale, la question de l'éventuel effet renonciateur d'une clause de compétence à une offre générale d'arbitrage ne se pose plus. Les deux contentieux se trouvent nettement séparés, du moins lorsqu'aucune clause d'electa una via n'est susceptible de les relier à nouveau. Mais, dans cette dernière hypothèse, la clause de compétence insérée dans le contrat n'interfère pas réellement avec la mise en œuvre du principe conventionnel d'electa una via. C'est la seule et effective saisine du juge interne de l'État d'accueil qui gouverne sa mise en œuvre, qu'elle le soit ou non sur le fondement d'une clause attributive de compétence est sans importance.

\section{Option contentieuse issue d'un même traité ou d'une même loi de protection des investissements étrangers}

Lorsqu'il estime avoir subi un préjudice imputable à l'État d'accueil, l'investisseur aura la possibilité de saisir le tribunal arbitral sur le fondement d'une offre générale d'arbitrage prévue au sein du TBI conclu entre ce dernier État et son État d'origine, ou éventuellement d'un traité multilatéral de même nature auquel sont parties ces deux États. Dans ce cas, l'investisseur bénéficiera le plus fréquemment d'un choix d'arbitrages multiples (1). Il pourra également être tenté d'élargir ces possibilités de choix procéduraux en ayant recours à la clause de la nation la plus favorisée (2).

\section{Clause d'arbitrages multiples}

Le plus souvent, outre la possibilité de saisir les juridictions locales, les offres générales d'arbitrage, posées à la faveur d'un traité ou d'une loi nationale, ouvrent à l'investisseur souhaitant engager une action à l'encontre de l'État d'accueil sur leurs fondements un choix entre plusieurs institutions ou règlements d'arbitrage. L'investisseur est alors mis en présence d'un choix entre une procédure CIRDI et une procédure devant une autre institution arbitrale, comme la CCI ou la LCIA, voire un arbitrage ad hoc en application du règlement de la CNUDCI.

SC du 14 nov. 2005, \148; Siemens A.G. c. Argentine, aff. n ARB/02/8, SC du 3 août 2004, \180, JDI 2005, p. 142, obs. E. Gaillard; AEs Corp. c. Argentine, aff. n ARB/02/17, SC du 26 avr. $2005, \int 50$. 
Pour prendre un exemple, l'article 8-2 du TBI conclu entre la France et l'Argentine, signé le 3 juillet 1991, prévoit qu'un différend n'ayant pu être réglé à l'amiable dans un délai de six mois peut être soumis «à la demande de l'investisseur» soit aux juridictions nationales de l'État partie impliqué dans le différend, «soit à l'arbitrage international». Dans cette dernière hypothèse, l'article 8-3 précise alors que l'investisseur peut à son choix porter le différend soit «devant le Centre international pour le règlement des différends relatifs aux investissements (CIRDI)», soit «devant un tribunal d'arbitrage $a d h o c »$, établi selon les règles drarbitrage de la CNUDCI. Selon la même technique tirée cette fois d'un traité multilatéral, l'article 26 du traité sur la Charte de l'énergie du 17 décembre $1994{ }^{19}$ prévoit que chaque État partie «donne son consentement inconditionnel à la soumission de tout différend à une procédure d'arbitrage», l'investisseur pouvant opter pour le CIRDI, un arbitrage ad hoc constitué sur la base du règlement de la CNUDCI ou une procédure d'arbitrage sous l'égide de l'Institut d'arbitrage de la Chambre de commerce de Stockholm.

En matière de loi nationale relative aux investissements étrangers, l'article 16 de la loi du Kosovo n 02/L-33 du 21 novembre 2005 sur cette question prévoit les dispositions suivantes: «A foreign investor shall have the right to require that the investment dispute be settled through arbitration in accordance with the procedural rules chosen by the foreign investor». Le texte poursuit en offrant un choix à l'investisseur entre le CIRDI, la CCI ou un arbitrage sur la base du règlement de la CNUDCI.

Le choix finalement opéré par l'investisseur sera gouverné par les divergences de régime existant entre ces différents types d'arbitrage. En pratique, ces différences les plus saillantes existent entre l'arbitrage CIRDI et les autres formes d'arbitrage proposées.

Du point de vue de l'établissement de la compétence, le choix d'un tribunal arbitral ne relevant pas du CIRDI pourrait s'avérer pertinent lorsque l'existence d'un investissement au sens de l'article 25 de la Convention de Washington du 18 mars 1965 fait débat. Un tel tribunal se contentera en principe de vérifier l'applicabilité de l'un ou l'autre des critères de l'investissement, tels que posés par le TBI ou le traité multilatéral sur le fondement duquel il a été saisi, lesquels définissent le plus souvent un champ d'application particulièrement large; il se dispensera le plus souvent

19 Sur ce texte, v. not. Energy Charter Treaty: An East-West Gateway for Investment and Trade, Th. Wälde (éd.), Kluwer Law International, 1996. 
d'opérer le fameux «double test» qu'impose la jurisprudence rendue sous les auspices du CIRDI. ${ }^{20}$

En faveur du choix d'une procédure arbitrale sous l'égide du CIRDI, en revanche, on relèvera notamment l'existence d'un régime particulièrement favorable à l'exécution de la sentence. En effet, dès lors qu'elle est prononcée sous les auspices de cette institution, celle-ci est exécutoire de plein droit sur le territoire de tous les États parties à la Convention de Washington de 1965, c'est-à-dire sans procédure d'exequatur préalable (Conv. CIRDI, art. 54). En outre, aucun appel ou recours en annulation de la sentence ne peut être porté devant le tribunal d'un État partie à la Convention de Washington —Conv. CIRDI, art. 53 (1) — . Les seules voies de recours possibles doivent être exercées dans le cadre du système du CIRDI, notamment le recours en annulation qui doit être porté devant un comité ad hoc (Conv. CIRDI, art. 52).

Si l'investisseur se prononce en faveur d'un arbitrage en dehors du CIRDI, on relèvera que la sentence une fois rendue sera susceptible de faire l'objet d'un contrôle de la part des juridictions internes, soit lors d'une procédure d'exequatur, soit même à l'occasion d'un recours en annulation. En réalité, elle suivra alors le même régime que les sentences rendues dans le cadre de l'arbitrage commercial international stricto sensu. Il est vrai que cette transposition à l'arbitrage en matière d'investissement des règles nationales applicables aux procédures d'exequatur ou aux recours en annulation propres aux sentences du commerce international pourrait être discutée. La nature commerciale d'une opération qui est en principe exclusive de celle d'investissement lors de la mise en œuvre de l'offre d'arbitrage réapparaît ainsi lors du contrôle de la sentence. Mais, en pratique, la mise en œuvre à l'égard de sentences rendues en matière d'investissement des règles internes propres à l'arbitrage commercial international se vérifie tant en droit français ${ }^{21}$ que dans de nombreux systèmes juridiques étrangers. ${ }^{22}$

20 Ceskoslovenska obchodni banka (CSOB) c. Slovaquie, aff. n ARB/97/4, ICSID Rev. 1999, p. 251, \ 68 .

21 La cour d'appel de Paris a eu à connaître d'un recours en annulation à l'encontre d'une sentence prononcée sur le fondement d'un TBI: CA Paris, 25 sept. 2008, Rép. tchèque c. Pren Nreka, D. 2009, p. 917, note M. Audit; RD aff. int. 2009, p. 409, obs. E. Cabrol; Gaz. Pal. 14-16 déc. 2008, p. 51, note B. Poulain; RGDI publ. 2008, p. 954, obs. Y. Nouvel.

22 On peut citer à cet égard la jurisprudence britannique (High Court of Justice, 29 avr. 2005, Republic of Ecuador v. Occidental Exploration and Production Co. [2005] EWCA Civ. 1116; [2006] QB 432 (CA)), canadienne (Supreme Court of British Columbia, 2 mai 2001, United 


\section{Recours au traitement de la nation la plus favorisée à des fins procédurales}

D'assez nombreux TBI octroient aux investisseurs ressortissants de chacun des États parties le traitement de la nation la plus favorisée. ${ }^{23}$ En priorité, c'est bien évidemment le traitement substantiel dont peuvent se prévaloir les investisseurs qui est visé par cette disposition. Mais la question s'est toutefois posée de l'extension de son champ d'application à l'ensemble des dispositions posées par les TBI, c'est-à-dire y compris au mécanisme de règlement des différends nés entre l'investisseur et l'État d'accueil. Si tel était le cas, un investisseur ressortissant d'un État avec lequel l'État d'accueil a conclu un TBI stipulant un tel traitement pourrait entendre se prévaloir d'un mécanisme de règlement des litiges jugé plus favorable, et prévu dans un autre TBI conclu par l'État d'accueil avec un État tiers. ${ }^{24}$

En jurisprudence, c'est à l'occasion de l'affaire Maffezini que, pour la première fois, un tribunal arbitral du CIRDI a été saisi d'une demande d'application du traitement de la nation la plus favorisée aux dispositions conventionnelles prises par un TBI en matière de règlement des litiges. ${ }^{25}$ En l'espèce, la requête avait été introduite à l'encontre de l'Espagne sur le fondement de l'offre d'arbitrage visée dans le TBI conclu entre cet État et

Mexican States v. Metalclad Co, 5 ICSID Rep. 236; Ontario Superior Court of Justice, 3 déc. 2003, United Mexican States v. Feldman Karpa, 8 ICSID Rep. 500; Federal Court of Canada, 13 janv. 2004, Attorney General of Canada v. S.D. Myers, 8 ICSID Rep. 194), des États-Unis (US District Court for the District of Columbia, 31 oct. 2005, 10 ICSID Rep. 448), suisse (T. fédéral, 10 nov. 2005, France Telecom c. Liban, aff. 4P.98/2005/svc.; T. fédéral, 7 sept. 2006, Saluka c. Tchéquie, aff. 4P.114/2006/bie) ou encore suédoise (Cұech Republic c. CME Czech Republic, aff. T8735-01, 9 ICSID Rep. 439).

23 Sur ce mécanisme, v. not. P. Daillier, M. Forteau et A. Pellet, Droit international public, 8e éd., Paris, LGDJ, 2002, n 158; Ann. CDI, 1978, vol. II, t. 1, pp. 19-81.

24 E. Gaillard, «Establishing Jurisdiction Through a Most-Favored-Nation-Clause», New York Law Journal 2005, p. 3; W. BEN HAMIDA, «Clause de la nation la plus favorisée et mécanismes de règlement des différends: que dit l'histoire ?", JDI 2007, p. 1126.

25 Emilio Agustin Maffezini c. Espagne, aff. n ARB/97/7, SC du 25 janv. 2000, ICSID Rev. 2001, p. 212. Sur cette affaire, v. not. B. Stern, «ICSID Arbitration and the State's Increasingly Remote Consent: A propos the Maffezini Case», in Law in the Service of Human Dignity, Essays in Honour of Florentini Feliciano, Cambridge, Cambridge University Press, 2005, pp. 246-260; V. Vicuña, «Bilateral Investment Treaties and the Most-Favored-Nation Clause: Implications for Arbitrations in the Light of a Recent ICSID Case», in Investment Treaties and Arbitration, G. Kaufmann-Kohler and B. Stucki (éd.), Bull. ASA, special series, 2002, pp. 133-144.

ACDI, Bogotá, ISSN: 2027-1131/ISSNe: 2145-4493, Vol. 10, pp. 333-370, 2017 
l'Argentine, laquelle imposait à l'investisseur, avant toute requête devant le CIRDI, une saisine préalable des juridictions locales ainsi qu'un délai de dix-huit mois afin de réserver à celles-ci un délai suffisant pour se prononcer sur le litige. Or, au titre du traitement de la nation la plus favorisée, l'investisseur a pris le parti d'invoquer le TBI conclu entre l'Espagne et le Chili, aux termes duquel seule une période de six mois de négociation devait s'être écoulée avant la saisine du CIRDI.

Dans cette affaire, le tribunal arbitral a entériné cette interprétation en reconnaissant l'applicabilité du traitement de la nation la plus favorisée en matière de règlement des différends. ${ }^{26}$ Toutefois, il a également posé des limites à cette applicabilité, en considérant que le bénéficiaire de la clause, c'est-à-dire l'investisseur, ne doit pas se prévaloir de celle-ci de manière à faire échec à des «considérations dıordre public» que les parties au TBI ont jugé essentielles lors de l'expression de leur consentement à celui-ci. ${ }^{27} \mathrm{Au}$ titre de telles considérations, la sentence mentionne l'épuisement des voies de recours, l'electa una via, le choix du CIRDI comme institution d'arbitrage ou l'intégration dans un système très intégré comme celui de l'ALENA. ${ }^{28}$

Dans des affaires postérieures, des tribunaux arbitraux saisis de cette question ont repris ce critère de l'ordre public comme limite à l'application du traitement de la nation la plus favorisée en matière de règlement des litiges. ${ }^{29}$ Dans d'autres sentences, il a en revanche été refusé d'étendre le bénéfice du traitement de la nation la plus favorisée aux clauses de règlement des différends. ${ }^{30}$ On en est donc réduit au constat que, sur cette question de l'applicabilité ou non du traitement de la nation la plus favorisée en matière de règlement des différends, la jurisprudence arbitrale n'est pas fixée.

\footnotetext{
26 SC préc. du 25 janv. 2000, \56.

27 Ibid., $\$ 62$.

28 Ibid., $\int$ 63. Pour un commentaire critique, C. Santulli, Droit du contentieux international, op. cit., $\mathrm{n}^{\circ} 231$.

29 Siemens AG c. Argentine, aff. n ARB/02/8, S. du 3 août 2004, \109, JDI 2005, p. 149, obs. E. Gaillard; National Grid c. Argentine, SC ad hoc du 20 juin 2006, \62; Suez, Sociedad General de Aguas de Barcelona S.A. and Vivendi Universal S. A. c. Argentine, aff. $\mathrm{n}^{\circ}$ ARB/03/19, SC du 3 août 2006, \62, Rev. arb. 2006, p. 1060, obs. M. Audit.

30 Plama Consortium Ltd. c. Bulgarie, aff. n ARB/03/24, SC du 8 févr. 2005, \$223, ICSID Rev. 2005, p. 262; Telenor Mobile Communications AS c. Hongrie, aff. n ARB/04/15, S du 13 sept. 2006, SS 98-100, ICSID Rev. 2006, p. 603, JDI 2007, p. 298, obs. E. Gaillard.
} 


\section{La concurrence des procedures contentieuses}

La variété d'options contentieuses ouvertes aux investisseurs laisse fréquemment apparaitre des situations où coexistent plusieurs procédures pour une même opération d'investissement. Ce phénomène est en outre accentué par le fait que, pour une même opération, il peut bien évidemment exister plusieurs investisseurs. Ce sera le cas, par exemple, lorsqu'il existe une société locale et une société mère étrangère, ou plus généralement une pluralité d'actionnaires lésés. Chacun d'entre eux est ainsi susceptible de retenir un choix procédural différent. À ceci, il faut ajouter que l'État d'accueil de l'investissement lui-même ou l'une de ses émanations partie à celui-ci est susceptible d'avoir pris, de son côté, l'initiative d'une procédure, notamment devant ses propres juridictions.

Dans ces conditions, il existe un risque important de voir prononcer des décisions contradictoires ou redondantes. Un même préjudice pourra ainsi être indemnisé plusieurs fois, ou le même type de préjudice affectant des investisseurs distincts différemment réparés, comme on avait pu déjà le constater en son temps avec les trois affaires dites libyennes dans les années soixante-dix. ${ }^{31} \mathrm{Il}$ y a là une source évidente de perturbation, voire de remise en cause possible, de l'ensemble du système arbitral en matière d'investissement. ${ }^{32}$ Un premier ensemble de solutions peut être trouvé en amont, c'est-à-dire au moment même où une concurrence procédurale se fait jour, en procédant à une consolidation des procédures contentieuses (A). Mais la faible mise en œuvre, pour l'heure, de ce type de mécanismes de regroupement des demandes en matière d'arbitrages relatifs aux investissements laisse apparaître des hypothèses de concurrence tant avec des procédures en cours (B) qu'avec des décisions antérieures $(C)$, pour lesquelles des solutions doivent être élaborées.

31 À la suite de la décision adoptée par l'État libyen de procéder à la nationalisation de l'ensemble de son industrie pétrolière, trois procédures arbitrales ont été diligentées à son encontre par des entreprises occidentales dépossédées, dont la plus emblématique qui a conduit à la sentence Texaco rendue en 1977 par R.-J. Dupuy au titre d'arbitre unique: Texaco Calasiatic c. Lybie, S. du 17 janv. 1977, AFDI 1977, p. 452. Pour les deux autres sentences: Liamco c. Lybie, S. du 12 avr. 1977, Rev. arb. 1980, p. 132; BP c. Lybie, S. 10 oct. 1973, Rev. arb. 1980, p. 117. Sur l'absence d'unité des solutions adoptées, v. B. Stern, «Trois arbitrages, un même problème, trois solutions: les nationalisations pétrolières libyennes devant l'arbitrage international», Rev. arb. 1980, pp. 3-43.

32 W. Ben Hamida, «L'arbitrage État-investisseur face à un désordre procédural: la concurrence des procédures et les conflits de juridictions», op. cit., pp. 564-602. 


\section{A. Consolidation des procédures contentieuses}

L'une des hypothèses de dispersion du contentieux en matière de protection des investissements est celle où, pour une même opération, plusieurs investisseurs introduisent des requêtes arbitrales distinctes, susceptibles de mener à la constitution de tribunaux arbitraux différents, eux-mêmes à même de délivrer des solutions divergentes à ce qui reste pourtant une même affaire lato sensu. ${ }^{33}$ Dans ce type d'hypothèses, certains tribunaux arbitraux sont susceptibles de disposer d'outils, encore un peu sommaires, pour y pallier. Ils peuvent en effet en opérer la consolidation. Mais si celle-ci est parfois institutionnellement organisée (1), elle peut également intervenir à l'initiative des parties (2).

\section{Consolidation institutionnellement organisée}

Il est bien évident que l'introduction de différentes procédures arbitrales, lorsqu'elles sont toutes reliées à une même opération d'investissement, n'est pas de nature à permettre une administration harmonieuse de la justice. La doctrine est donc tout à fait consciente qu'il peut s'avérer pertinent d'en opérer la consolidation devant un seul et même tribunal arbitral. ${ }^{34}$ Toutefois, pour l'heure, les systèmes d'arbitrage spécifiques à la protection des investissements, qui permettent la consolidation de procédures, sont peu nombreux.

Le plus connu et le plus ancien est celui qui résulte du chapitre 11 de l'ALENA. En présence de litiges présentant des questions de droit ou de fait communes, l'article 1126 de cet accord multilatéral permet en effet d'imposer aux parties un regroupement des demandes devant un seul et

33 Un tribunal arbitral fait le constat suivant: «The desirability of avoiding conflicting results is not limited to cases where the parties are the same. Cases with different parties may present the same legal issues arising out of the same event or related to the same measure. Conflicting results then may take place if the findings with respect to those issues differ in two or more cases». (Canfor Corporation v. United States of America, Tembec et al. $v$. United States of America, and Terminal Forest Products Ltd. $v$. United States of America, Order of the Consolidation Tribunal, 7 sept. 2005, \ 133).

34 W. Ben Hamida, «La consolidation des procédures arbitrales», Ga»: Pal. 13-14 déc. 2006, p. 30; G. Kaufmann-Kohler, L. Boisson de Chazournes, V. Bonnin and M. M. Mbengue, «Consolidation of Proceedings in Investment Arbitration: How Can Multiple Proceedings Arising from the Same or Related Situations be Handled Efficiently ?», ICSID Rev. 2006, pp. $59-125$. 
même tribunal arbitral. ${ }^{35}$ La décision doit toutefois être prise «dans l'intérêt d'un règlement juste et efficace des plaintes, et après audition des parties contestantes $»{ }^{36}$ Le modèle 2004 de TBI préconisé par les États-Unis a du reste repris cette possibilité de consolidation, en adoptant une règle très proche. Son article 33, alinéa 1er, prévoit en effet la disposition suivante: «Where two or more claims have been submitted separately to arbitration under Article 24(1) and the claims have a question of law or fact in common and arise out of the same events or circumstances, any disputing party may seek a consolidation order in accordance with the agreement of all the disputing parties sought to be covered by the order or the terms of paragraphs 2 through $10 »$. À l'appui d'un mécanisme très élaboré, l'article 33 détaille ensuite le processus de consolidation dont il confie la supervision au Secrétaire général du CIRDI.

Mais pour autant, de son côté, le système du CIRDI ne prévoit pas de disposition spécifique du même type susceptible de s'appliquer d'une manière générale. Dès lors, dans la plupart des contentieux arbitraux relatifs à l'investissement étranger, il n'existe pas de règles permettant d'imposer aux parties un regroupement de procédures. C'est assurément là une carence du système, parfois constatée par les tribunaux arbitraux eux-mêmes ${ }^{37}$ et dont on espère qu'elle puisse à terme être un jour définitivement comblée.

La nécessité d'une consolidation des procédures est du reste parfois tellement flagrante qu'elle est entreprise en dehors de toute disposition spécifique prévue par le règlement d'arbitrage applicable. Elle intervient alors à l'initiative des parties ou du tribunal arbitral lui-même.

35 Pour un ex., Canfor Corporation v. United States of America, Tembec et al. v. United States of America, and Terminal Forest Products Ltd. $v$. United States of America, Order of the Consolidation Tribunal, 7 sept. 2005. Sur cette procédure, v. not. B. Hanotiau, «NAFTA Consolidation Decision Under Art. 1126 of the NAFTA», Transnational Dispute Management November 2005, vol. 2 , issue 05 .

36 L'article 34 du projet d'accord de zone de libre-échange des Amériques (ZLELA), dont l'objectif est d'étendre la zone de libre-échange mise en place par l'ALENA à toute l'Amérique latine, prévoit également un mécanisme de jonction de procédures arbitrales du même type.

37 «Le Centre a fait tous les efforts possibles pour éviter une multiplicité de tribunaux et de compétences, mais il n'est pas possible d'empêcher différents investisseurs d'exercer les droits qu'ils sont susceptibles d'avoir en vertu de différents instruments», CMS Gas Transmission Company c. Argentine, aff. n ARB/01/8, SC du 17 juill. 2003, S 85, ILM 2003, p. 78, JDI 2004, pp. 236-257 (pour la trad. française), obs. E. Gaillard. 


\section{Consolidation à l'initiative des parties ou du tribunal arbitral}

Face à l'inexistence, en dehors du chapitre 11 de l'ALENA, d'un système institutionnellement organisé de consolidation des procédures arbitrales en matière d'investissements, il peut arriver qu'elle soit opérée en dehors de tout cadre préexistant, notamment lorsque plusieurs investisseurs introduisent une requête arbitrale à l'encontre d'un même État défendeur sur le fondement d'un même instrument et pour les mêmes faits.

C'est l'hypothèse par exemple d'actionnaires d'une même société locale introduisant une telle requête conjointement à l'encontre de l'État d'accueil sur le fondement d'un TBI conclu avec leur État d'origine. Plus rarement, sont également opérés des regroupements de demandes fondées — cette fois-ci - sur des instruments distincts, contrat et TBI notamment. Certains tribunaux arbitraux ont en effet entériné de telles consolidations de procédures, non sans avoir au préalable vérifié l'interdépendance des demandes et la pertinence de les voir jugées au cours d'une même instance. ${ }^{38}$

Comme elles sont mises en œuvre en dehors de toute disposition qui les prévoiraient dans le règlement d'arbitrage applicable, la question du fondement et du régime de ces décisions de consolidation se pose. Plus précisément, il s'agit de savoir si le tribunal arbitral a la possibilité de l'imposer sur le seul fondement du pouvoir d'organisation de l'instance d'arbitrage qui est le sien, ou s'il doit nécessairement recueillir le consentement, même implicite, de l'ensemble des parties concernées, notamment les parties étatiques. En l'état de la jurisprudence comme de la réflexion doctrinale, ce point n'est toujours pas définitivement tranché. ${ }^{39}$

\section{B. Concurrence avec une procédure en cours}

Les mécanismes de consolidation des procédures étant, pour l'heure, sommaires en matière de litiges relatifs à la protection des investissements étrangers, il est finalement fréquent que plusieurs instances soient saisies

\footnotetext{
38 Noble Energy Inc. and MachalaPower Cía. Ltd. c. Équateur et Consejo Nacional de Electricidad, aff. $\mathrm{n}^{\circ}$ ARB/05/12, SC du 5 mars 2008; Occidental Petroleum Corp. and Occidental Exploration and Production Co. c. Équateur, aff. n ARB/06/11, S. du 9 sept. 2008.

39 K. Yannaca-Small, "Parallel Proceedings», in The Oxford Handbook of International Investment Law, P. Muchlinski, F. Ortino and Ch. Schreuer (eds), Oxford University Press, Oxford, 2008, pp. 1009-1048, spéc. pp. 1042-1043.
} 
d'une même affaire, ou d'affaires proches et relatives à une même opération d'investissement.

Pour y remédier, les tribunaux arbitraux sont allés puiser dans les ressources conceptuelles du droit processuel. Nombre d'entre eux ont ainsi convoqué le principe bien connu de lis alibi pendens, mieux connu sous le nom de litispendance (1). Mais, comme les conditions nécessaires à la mise en œuvre de cette exception sont traditionnellement considérées comme très restrictives, la question de l'admission, à son côté, de l'exception de connexité est parfois posée (2).

\section{Litispendance (Lis pendens)}

L'exception de litispendance est bien connue du contentieux étatique. Elle impose ou, en tout état de cause, permet à un tribunal saisi en second de se dessaisir en présence d'une instance plus ancienne portant sur le même litige. Or, en matière d'arbitrage relatif aux investissements, ce type d'exception procédurale est parfois invoqué lorsqu'il existe une instance en cours relative à la même opération d'investissement au moment de l'introduction de la requête. En pratique, on constate néanmoins que, le plus souvent, les tribunaux arbitraux saisis d'une telle demande rejettent l'exception de litispendance. À cet effet, ils relèvent que la triple identité — parties, cause et objet — à laquelle on soumet traditionnellement la litispendance en droit processuel n'est pas réunie. ${ }^{40}$

Or, de ce point de vue, il est vrai que le plus souvent l'instance concurrente ne réunit pas exactement les mêmes parties, en ce sens que l'investisseur demandeur sera différent, même si l'opération elle-même et le comportement reproché à l'État sont alors identiques. Dans une telle situation, et à retenir la conception traditionnelle de la litispendance, on ne pourra à l'évidence retenir une identité de parties entre les deux procédures. C'est une consolidation des procédures qui aurait ici été indiquée, mais, comme on a pu le constater, ${ }^{41}$ ce mécanisme est encore très embryonnaire en matière de litiges arbitraux relatifs à la protection des investissements étrangers.

40 SGS c. Pakistan, aff. $\mathrm{n}^{\circ}$ ARB/01/13, SC du 27 août 2003, \ 182, JDI 2004, p. 257, obs.

E. Gaillard; Lauder c. Rép. tchèque, S. préc. ad hoc du 3 sept. 2001, \$171; Benvenuti et Bonfant c. Congo, aff. nº ARB/77/2, S. du 8 août 1980, \1.14, ICSID Rep. 1993, p. 330.

41 Supra, pp. 366-368. 
Cela étant, cette conception stricte de l'identité de parties conduit à pouvoir considérer que la requête arbitrale introduite par une société est distincte de celle qu'ont diligentée ses actionnaires. Formellement, les parties sont alors effectivement différentes, mais il n'en reste pas moins qu'il s'agit de la même opération d'investissement, et du même comportement qui est reproché à l'État, voire du même TBI fondant la compétence arbitrale. ${ }^{42}$

Du reste, même lorsque, tant du côté de l'investisseur que du côté étatique, les deux parties aux procédures concomitantes sont identiques, les conditions de la litispendance ne sont pas pour autant nécessairement réunies. C'est alors l'identité d'objet ou de cause qui font défaut.

Ainsi, lorsqu'il s'agit de deux procédures arbitrales opposant les mêmes parties, cela s'explique bien souvent par le constat que l'une d'entre elles est fondée sur une clause compromissoire insérée dans un contrat conclu avec l'État, tandis que l'autre a été initiée par l'investisseur en application d'une offre d'arbitrage insérée dans un traité de protection des investissements. Autrement dit, on est en présence d'un contract claim dans un cas et d'un treaty claim dans l'autre; les tribunaux arbitraux vont pouvoir en déduire une absence d'identité de cause entre les deux procédures, exclusive de toute litispendance. ${ }^{43}$

C'est cette absence d'identité de cause qui explique également le peu, voire l'absence, d'incidence de l'exception de litispendance en présence d'une coexistence entre une procédure arbitrale internationale et une procédure devant une juridiction nationale ${ }^{44}$ ou une juridiction supranationale permanente. Dans l'affaire Eureko c. Slovaquie, l'investisseur avait saisi ainsi la Commission européenne qui a introduit une action en manquement à l'encontre de cet État, alors même qu'une procédure arbitrale a également

42 Sur ce point, v. l'arrêt prononcé par une cour d'appel suédoise à propos des affaires ayant opposé M. Lauder et la société CME à la République tchèque: Svea Cour of Appeal, 15 may 2003, Czech Republic v. CME Czech Republic BV, Case No. T 8735-01, cité par N. Gallagher, «Parallel Proceedings: res judicata and lis pendens in Contemporary Problems», in International Arbitration, Lew \& Mistelis (eds), Kluwer Law International, 2006, pp. 329-356, spéc. p. 343.

43 Compañia de Aguas del Aconquija and Vivendi Universal S.A. c. Argentina, aff. n ARB/97/3, DCAH préc. du 3 juill. 2002, \$S 102 et 103.

44 Y. Shany, «Jurisdictional Competition Between National and International Courts: Could International Jurisdiction-Regulating Rules Apply ?», Netherlands Yearb. Int. L. 2006, pp. 3-56; W. S. Dodge, «National Courts and International Arbitration: Exhaustion of Remedies and Res Judicata under Chapter Eleven of NAFTA», Hastings Int. and Comp. L. Rev. 2000 , p. 357. 
été diligentée par ses soins sur le fondement du TBI conclu en 1992 entre les Pays-Bas et la Tchécoslovaquie. ${ }^{45}$ Quant à la saisine concomitante de la Cour européenne des droits de l'homme et de tribunaux arbitraux, l'affaire Yukos en est un exemple emblématique, ${ }^{46}$ même si elle n'est pas isolée. ${ }^{47}$

Mais que l'instance parallèle ait été portée devant un tribunal étatique ou devant une juridiction supranationale de nature permanente, le constat est identique: entre les deux procédures, la cause de l'action est distincte. Devant un juge interne, ce sont le plus souvent des dispositions du droit national qui fondent l'action. ${ }^{48}$ Quant aux instances devant la Cour de Strasbourg, en lien avec une opération d'investissement, elles s'appuient généralement sur une méconnaissance — supposée à ce stade — par l'État d'accueil du droit de propriété de l'investisseur, tel que protégé par l'article $1 \mathrm{er}$ du Protocole $\mathrm{n}^{\circ} 1$ de la Convention européenne des droits de l'homme. Or, s'agissant maintenant de l'instance arbitrale, elle est fondée sur une méconnaissance soit d'un traité de protection des investissements, soit d'un contrat en lien avec le droit qui lui est applicable.

En bref, dans l'immense majorité des hypothèses, lorsque ce n'est pas l'identité de parties, ce sera l'identité de cause, voire d'objet, nécessaire à la mise en œuvre de l'exception de litispendance, qui ne sera pas vérifiée. Ce constat, pour le moins déceptif, conduit à douter de la pertinence du

45 Eureko B.V.c. Rép. de Slovaquie, CPA n² 2008-13, S. du 26 oct. 2010, Rev. arb. 2011, p. 247, note B. Poulain. L'instance européenne est en revanche toujours en cours.

46 Hulley Enterprises Limited (Cyprus) c. Russie, Yukos Universal Limited (Isle of Man) c. Russie et Veteran Petroleum Limited (Cyprus) c. Russie, CPA n ${ }^{\circ}$ AA 226, AA 227 et AA 228, 3 SC du 30 novembre 2009.

47 Pour d'autres affaires où la Cour européenne des droits de l'homme a été saisie par l'investisseur, ou l'une de ses entités, concomitamment à la procédure arbitrale: Saluka Investments B.V. c. République tchèque, S . part. CPA du 17 mars 2006; Amto c. Ukraine, SCC 080/2005, S. du 26 mars 2008. Il peut également arriver que l'investisseur renonce finalement à saisir la Cour de Strasbourg: L. Peterson, «Italians Drop Human Rights Claim Against Georgia, Initiate Investment Treaty Claim», Investment Treaty News 29 mars 2006. Sur cette question, v. not. W. Ben Hamida, «Investment Arbitration and Human Rights», TDM Sept. 2007, vol. 4, issue 5, p. 11.

48 Un auteur opère ainsi le constat suivant: «Dans la mesure où les deux procédures ne contrôlent pas les mêmes normes, l'investisseur peut recourir aux deux mécanismes. Il peut saisir le juge interne pour contester les violations du droit interne. Il peut aussi simultanément ou successivement saisir un tribunal arbitral statuant sur le fondement d'un traité international d'investissement pour contester les violations du droit international». W. Ben Hamida, «L'arbitrage État-investisseur face à un désordre procédural: la concurrence des procédures et les conflits de juridictions», op. cit., p. 571. 
concept de litispendance en matière de contentieux arbitral relatifs à la protection des investissements étrangers. ${ }^{49} \mathrm{Il}$ est vrai que, finalement, en raison de cette mise en œuvre sourcilleuse de la triple identité, les tribunaux arbitraux ne s'estiment pas contraints de se dessaisir sur son fondement. ${ }^{50}$ Ceci explique qu'on ait pu proposer d'en adopter une formulation plus souple et teintée de considérations plus économiques que juridiques. Ce serait l'unité économique du litige qui devrait être prise en considération, ce qui permettrait notamment de réunir actionnaires et la société en tant que victime directe, et la mesure reprochée à l'État défendeur devrait seule être retenue comme cause du litige, ce qui aurait pour effet de se départir d'un certain compartimentage entre procédures fondées sur des instruments distincts. ${ }^{51}$

Mais la jurisprudence arbitrale n'est pas généralement sensible à cette version renouvelée de l'autorité négative de chose jugée. ${ }^{52}$

Mais, en contrepoint de ce dernier constat, les difficultés nées de la coexistence de deux procédures parallèles ne trouvent pas alors de solution satisfaisante, ce qui pourrait conduire à l'émergence d'une exception de connexité, sans doute bien plus efficiente en ce domaine.

\section{Débat sur l'avènement de la connexité}

La matière du droit des investissements internationaux connaît un réel problème de parallélisme procédural, en ce sens que le risque de voir se développer des solutions distinctes, voire opposées, pour un même litige ou des litiges liés à une même opération d'investissement est très prégnant. Pourtant, il apparaît évident que l'exception de litispendance, en tout cas dans sa conception la plus traditionnelle fondée sur le constat d'une triple identité, n'est pas à même de régler ce type de difficulté. En pratique, on constate que la litispendance est souvent invoquée, alors toujours discutée

49 G. Cuniberti, «Parallel Litigation and Foreign Investment Dispute Settlement», ICSID Rev. 2006, pp. 381-426.

50 En revanche, il peut arriver que le tribunal arbitral se prononçant en premier lieu enjoigne aux parties de se désister des autres procédures visant à réparer le même préjudice: Occidental Exploration and Production company c. Équateur, S. LCIA, du 1er juill. 2004, \$209.

51 A. Crivellaro, «Consolidation of Arbitral and Court Proceedings in Investment Disputes», The Law and Practice of International Courts and Tribunals 2005, p. 373.

52 V. not. CME c. Rép. tchèque, S. ad hoc du 13 sept. 2001, \ 436. 
dans la motivation des sentences, mais en dernière analyse pratiquement jamais effectivement retenue.

Certains auteurs comme certains panels arbitraux ont pris conscience de cette difficulté et c'est ainsi qu'a pu commencer à émerger une position plus flexible en matière de règlement des procédures parallèles. À cette fin, on a ainsi pu proposer de puiser à nouveau dans les catégories du droit processuel, mais pour en extraire - cette fois-ci — ce concept cousin de la litispendance que constitue la connexité. ${ }^{53}$ La grande différence entre ces deux notions est que la seconde d'entre elles ne repose pas sur la triple identité de parties, de cause et d'objet. Dès lors, sa mise en œuvre plus fréquente pourrait effectivement permettre une gestion plus souple de ce problème récurrent que constituent les procédures parallèles.

En présence de deux instances présentant un tel lien de connexité, en ce sens que la solution à venir dans l'une est susceptible d'avoir une incidence directe sur l'autre, le tribunal arbitral second saisi aurait la faculté, après analyse et sans y être contraint, de surseoir à statuer dans l'attente de la décision de la juridiction première saisie. ${ }^{54}$ Cette admission en la matière de la connexité aurait notamment comme avantage d'éviter que ne se rencontrent, par la suite, des problèmes d'autorité de la chose jugée.

\section{Concurrence avec une décision antérieure}

Outre la concurrence avec une autre instance en cours, un tribunal arbitral saisi en matière d'investissements est également susceptible d'être confronté à l'existence d'une décision antérieure et définitive rendue dans la même affaire, ou en lien avec celle qui lui a été confiée. Là encore, les ressources du droit processuel permettent d'avoir recours à la notion d'autorité de chose jugée. On sait que le principe de droit romain Res judicata pro veritate habetur fait l'objet, sous une forme ou sous une autre, d'une admission très large en droit comparé. ${ }^{55}$ À la vérité, on imagine mal qu'un système judiciaire,

53 P. Mayer, «Litispendance, connexité et chose jugée dans l'arbitrage international», in Liber Amicorum Claude Reymond. Autour de l'arbitrage, LexisNexis Litec, Paris, 2004, p. 185.

54 Southern Pacific Properties (Middle East) Limited c. Égypte, aff. n ARB/84/3, SC du 27 nov. 1985, \27; Société Générale de Surveillance S.A. c. Philippines, aff. $\mathrm{n}^{\circ} \mathrm{ARB} / 02 / 06, \mathrm{SC} \mathrm{du}$ 29 janv. 2004, ICSID Rep. 2005, p. 518, \ 175.

55 Sur ce point, v. L'étendue de l'autorité de la chose jugée en droit comparé, Étude réalisée par l'Institut de Droit comparé Édouard Lambert de l'Université Jean Moulin - Lyon 3: [http:/ / 
quel qu'il soit, puisse ne pas être doté d'un concept en application duquel les procès ne peuvent être indéfiniment recommencés.

Le système de règlement des conflits propres au droit international public est également confronté à cette même nécessité. Du reste, le principe d'autorité de chose jugée y est considéré comme un principe général de droit international au sens de l'article 38 du statut de la CIJ. ${ }^{56}$ Dans les litiges spécifiques à la protection des investissements étrangers, il est également admis par principe que les tribunaux arbitraux ne sauraient juger à nouveau des litiges qui ont d'ores et déjà connu un règlement définitif.

Du reste, on y rencontre même le concept d'autorité de chose jugée sous un double aspect. ${ }^{57}$ Aux termes du premier d'entre eux, l'existence d'une décision antérieure pourrait conduire le tribunal arbitral saisi en second lieu à se déclarer incompétent; c'est l'effet négatif de chose jugée (1). Le second effet ne le contraint pas au dessaisissement, mais il lui imposerait de tenir pour acquis ce qui a été jugé dans la décision antérieure; on parle alors d'effet positif (2).

\section{Effet négatif de chose jugée}

L'existence d'une décision antérieure rendue dans la même affaire pourrait contraindre un tribunal arbitral nouvellement saisi à se déclarer incompétent sur le fondement de l'autorité négative de chose jugée. En pratique, on constate néanmoins que les tribunaux arbitraux saisis d'une telle exception se refusent à l'accueillir. Les motifs invoqués sont identiques à ceux qui conduisent au rejet de la litispendance. Comme pour celle-ci, les tribunaux arbitraux rejettent les exceptions d'autorité négative de chose jugée dont ils sont saisis, la triple identité propre à celle-ci, c'est-à-dire de parties, d'objet et de cause, n'étant pas réunie. ${ }^{58}$

www.courdecassation.fr/IMG/File/Plen-06-07-07-0410672-rapport-definitif-anonymiseannexe-2.pdf/].

56 CPJI, avis consultatif du 16 mai 1975, Service postal polonais à Dantrig, Série B, nº 11, Rec., spéc. pp. 28-30. V. ég. Commission des réclamations France-Venezuela, Compagnie Générale de l'Orénoque, (1905) Ralston's Report, p. 244, spéc. p. 357; American-British Claims Tribunal (1921), S.S. Newchang, Claim No. 21, AJIL 1922, p. 323, spéc. p. 324.

57 K. Yannaca-Small, «Parallel Proceedings», op. cit., p. 1015.

58 CME c. Rép. tchèque, S. ad hoc du 14 mars 2003, \ 435, 9 ICSID Rep. 264. 
Il en est assurément ainsi lorsque la décision antérieure invoquée à l'appui de l'exception a été prononcée par un tribunal interne; ${ }^{59}$ l'identité d'objet ou de cause fait alors assurément défaut. Mais l'exception d'autorité négative de chose jugée a pu également être écartée en présence d'une sentence prononcée par un autre tribunal arbitral et ce, pour le même litige, dès lors que le nouveau tribunal saisi l'a été sur le fondement d'un instrument distinct, en d'autres termes d'une cause différente. Dans l'affaire CME c. République tchèque, le tribunal arbitral a opéré ce constat en des termes particulièrement nets: "The fact that one tribunal is competent to resolve the dispute brought before it does not necessarily affect the authority of another tribunal, constituted under a different agreement, to resolve a dispute - even if it were the "same" dispute». ${ }^{60}$

Autrement dit, si le premier tribunal arbitral a été saisi sur le fondement d'un contrat ou d'une loi nationale de protection des investissements, tandis que le second l'a été en application d'un TBI, l'autorité négative de chose jugée ne sera pas retenue, les parties et l'objet du litige fussent-ils identiques. Seul un fondement exactement identique à la saisine des deux tribunaux arbitraux successifs pourrait éventuellement conduire le second d'entre eux à se déclarer incompétent en application de l'effet négatif de l'autorité de chose jugée, ce qui limite assez considérablement ses occurrences.

Mais, même dans cette hypothèse, on constate que les tribunaux arbitraux saisis en second se montrent souvent réticents à donner son plein effet au principe de l'autorité négative de chose jugée. Ainsi, dans le litige ayant opposé la société américaine Waste Management à l'État du Mexique, une première requête arbitrale avait été introduite en application du chapitre 11 de l'ALENA. Ce premier tribunal arbitral a toutefois estimé que la condition à l'admission de la requête arbitrale, posée par l'article

59 Amco Asia Corporation c. Indonésie, aff. n ARB/81/1, SC du 25 sept. 1983, spéc. \S 50-52, Rev. arb. 1985, p. 259, JDI 1986, p. 202; Enron Corporation and Ponderosa Assets, L.P. c. Argentine, aff. $\mathrm{n}^{\circ} \mathrm{ARB} / 01 / 3$, S. du 14 janv. 2004, \$ 98; Occidental Exploration and Production Company c. Équateur, S. préc. du 1er juill. 2004, \ 60 et 61. Sur cette question dans le cadre spécifique du chap. 11 de l'ALENA: W. S. Dodge, «National Courts and International Arbitration: Exhaustion of Remedies and Res Judicata under Chapter Eleven of NAFTA», op. cit., p. 357.

60 CME c. Rép. tchèque, S. préc. du 14 mars 2003, \435. V. ég. Southern Pacific Properties (Middle East) Limited c. Égypte, aff. n ARB/84/3, SC du 27 nov. 1985, \27, ICSID Rev. 1993, p. 332; JDI 1994, p. 218, obs. E. Gaillard. 
1121-2, b, de l'Accord et imposant la renonciation à toute procédure juridictionnelle interne par l'investisseur, n'était pas en l'espèce remplie. Il s'est en conséquence estimé incompétent. ${ }^{61}$

À la suite de cette première sentence, l'investisseur s'est effectivement désisté de toutes ses procédures internes et a alors introduit une nouvelle requête arbitrale exactement sur le même fondement. La question de l'autorité négative de chose jugée s'est donc posée de manière frontale au second tribunal arbitral. Mais s'il reconnait sans difficulté la portée de la règle de res judicata dans son principe, ${ }^{62}$ il a en revanche estimé que l'investisseur pouvait prétendre réintroduire en l'espèce une nouvelle requête arbitrale, au motif essentiel que l'article 1121 du Traité de l'ALENA ne s'y opposait pas. ${ }^{63}$

On doit sans doute pouvoir en déduire que, sauf disposition contraire applicable, une première sentence d'incompétence rendue entre les mêmes parties ne permet pas de rejeter une seconde requête arbitrale, dès lors bien évidemment que le vice de compétence a été vidé. C'est donc finalement uniquement si la première sentence s'est prononcée sur le fond que l'autorité négative de chose jugée peut pleinement produire ses effets, ce qui limite à très peu d'hypothèses ses occurrences. Son effet positif pourrait en revanche être plus fréquemment accueilli.

\section{Effet positif de chose jugée}

De la manière la plus générale, l'autorité positive de chose jugée s'oppose à ce qu'une juridiction, faisant l'objet d'une saisine, remette en cause les constatations effectuées par une autre juridiction ayant rendu une précédente décision entre les mêmes parties à l'occasion d'une autre demande. La doctrine évoque alors l'«autorité positive de chose jugée» ou l'«effet positif d'autorité de chose jugée». ${ }^{64} \mathrm{Il}$ ne s'agit plus ici de s'opposer à un

61 Waste Management, Inc. v. United Mexican States, ARB(AF)/98/2, DC du 2 juin 2000, ILM 2001, vol. 40, p. 56, ICSID Rev. 2000, p. 211.

62 Waste Management, Inc. c. Mexique ( $\left.n^{\circ} 2\right), \mathrm{ARB}(\mathrm{AF}) / 00 / 3$, Mexico's Preliminary Objection concerning the Previous Proceedings, décision du Tribunal du 26 juin 2002, spéc. \47, ICSID Rep. 2004, p. 549.

63 Dans le même sens, Methanex Corporation v. United States of America, Memorial on Jurisdiction and Admissibility of Respondent United States of America, 13 November 2000, p. 77.

64 V. l'étude fondamentale du Doyen Héron sur ce concept en procédure civile française, 
nouveau procès — les demandes étant distinctes, il n'y a pas d'autorité négative de chose jugée —-, mais de tenir pour définitivement acquis ce qui a déjà été précédemment, et définitivement, jugé. L'autorité positive de chose jugée accélère le règlement du contentieux, en permettant à la juridiction seconde saisie de se concentrer sur les points de droit nouveaux entre les parties; elle permet également d'éviter que soient rendues des décisions contradictoires entre les mêmes parties.

Or, le contentieux pour une même opération d'investissement transnational étant très fréquemment plural, la question de l'accueil, ou non, de l'autorité positive de chose jugée se pose irrémédiablement. Les tribunaux arbitraux s'interrogent quant à déterminer s'il leur appartient de tenir pour acquis ce qui a été précédemment jugé devant un autre juge pour la même affaire ou un litige connexe.

Bien évidemment, lorsque les deux instances s'inscrivent dans le cadre d'une même procédure arbitrale, la réponse ne fait alors guère de doute. On vise en fait ici l'hypothèse où une première sentence — partielle par définition - a été prononcée par le même tribunal arbitral dans la même procédure. Cette première décision simpose ensuite au tribunal lui-même comme aux parties. En słappuyant à la fois sur les termes du TBI fondant sa compétence et sur l'article 32 (1) du règlement d'arbitrage de la CNUDCI, un tribunal arbitral a ainsi expressément énoncé le motif suivant: "The Tribunal's considered conclusion is that the Partial Award is binding upon the Tribunal and the parties». ${ }^{65}$ L'existence de ce principe de l'effet positif de chose jugée attaché à des sentences antérieurement rendues dans une même procédure est d'ailleurs assez fréquemment relevée. ${ }^{66}$ Seule la preuve d'une fraude pourrait être de nature à permettre aux arbitres de réexaminer le contenu de la sentence partielle et de revenir ainsi sur son caractère obligatoire et définitif. ${ }^{67}$

L'admission du principe de l'autorité positive de chose jugée est, en revanche, plus délicate lorsque les procédures sont différentes. Les tri-

J. Heron, «Localisation de l'autorité de chose jugée ou rejet de l'autorité positive de chose jugée ?», in Nouveaux juges, nouveaux pouvoirs? Mélanges en l'bonneur de Roger Perrot, Dalloz, Paris, 1996, p. 137.

65 CME c. Rép. tchèque, S. préc. du 14 mars 2003, \ 424.

66 Amco Asia Corporation c. Indonésie, aff. n ARB/81/1, SC du 10 mai 1988, \30, ICSID Rev. 1988, p. 166; JDI 1989, p. 143, obs. E. Gaillard; Plama Consortium Ltd. c. Bulgarie, aff. $n^{\circ}$ ARB/03/24, S. du 8 févr.2005, SS 180 et 18, ICSID Rev. 2005, p. 262.

67 Biloune et al. c. Ghana, S. du 30 juin 1990, ILR, vol. 95, pp. 211-232, spéc. pp. 222 et 223. 
bunaux arbitraux peuvent alors se montrer réticents à tenir pour acquises et incontestables les constatations tirées d'une précédente décision issue d'une procédure distincte de celle dont ils sont saisis, et par définition prononcée par un panel arbitral différemment composé. Par exemple, dans l'affaire dite du Platean des Pyramides, le tribunal CiRDI a refusé de tenir pour acquises certaines conclusions établies par une sentence CCI prononcée antérieurement. ${ }^{68}$

Dans une affaire plus récente, l'autorité positive de chose jugée a toutefois été admise, au moins dans son principe. Il s'agit du contentieux ayant opposé la société américaine Waste Management à l'État du Mexique. En effet, les motifs énoncés sur le fond par le premier tribunal arbitral, à l'occasion de sa sentence d'incompétence, ${ }^{69}$ ont été reconnus comme susceptibles en principe d'être dotés d'autorité — positive donc — de chose jugée dans le cadre de la seconde procédure arbitrale. Le tribunal s'y est exprimé dans les termes suivants: «The present Tribunal in no way denies the value of the principle of res judicata, nor its potential application in the present proceedings to the extent that any issue already decided between the parties may prove to be relevant at a later stage». ${ }^{70}$

Pour autant, pendant longtemps, les seuls effets véritablement entérinés par la jurisprudence arbitrale de l'autorité positive de chose jugée ont été limités à une question bien particulière: celle de l'indemnisation du préjudice de l'investisseur. Spécifiquement sur ce point, la prise en compte d'une décision antérieure a été en effet assez fréquemment admise. Il est vrai que la concurrence de procédures ne doit pas permettre qu'un même préjudice, fût-il évalué de manière différente et éventuellement à l'aune de règles de droit d'origine distincte, puisse faire l'objet de plusieurs indemnisations. ${ }^{71}$ Pour l'éviter, certains tribunaux arbitraux prennent en considération l'indemnisation d'ores et déjà obtenue par la victime dans le calcul de leur propre condamnation. ${ }^{72}$

68 Southern Pacific Properties (Middle East) Limited c. Égypte, aff. n ARB/84/3, SC préc. du 27 nov. 1985, \$27. Pour la sentence CCI rendue dans cette affaire: Southern Pacific Properties (SPP) c. Égypte, S. du 11 mars 1983, ILR, vol. 86, pp. 435-474.

69 Waste Management, Inc. v. United Mexican States, ARB(AF)/98/2, DC préc. du 2 juin 2000.

70 Waste Management, Inc. c. Mexique (n² 2), ARB(AF)/00/3, Décision préc. du 26 juin 2002, spéc. $\ 47$.

71 C. Santulli, Droit du contentieux international, op. cit., $\mathrm{n}^{\circ} 167$.

72 Lauder c. Rép. tchèque, S. ad hoc du 3 sept. 2001, \172; CME c. Rép. tchèque, S. ad hoc du 13 sept. 2001, \410; Wena c. Égypte, S. du 22 déc. 2000, \127, ILM 2002, p. 896. Sur cette 
Mais récemment, dans l'affaire Rachel S. Grynberg, Stephen M. Grynberg, Miriam Z. Grynberg, and RSM Production Corporation and others v. Grenada, un pas en avant très significatif a été fait s'agissant de la généralisation de l'autorité positive de chose jugée en matière de protection des investissements étrangers. ${ }^{73}$ Plus exactement, c'est la doctrine du collateral estoppel directement tirée de la common law qui a été entérinée par le tribunal arbitral dans les termes suivants: "The doctrine of is now well established as a general principle of law applicable in the international courts and tribunals such as this one». ${ }^{74} \mathrm{Or}$, le collateral estoppel produit des effets très proches de l'autorité positive de chose jugée, telle que dogmatisée dans les systèmes de tradition romano-germanique.

Dans cette affaire, la précédente sentence avait été rendue par un tribunal du CIRDI sur le fond, mais elle était fondée sur un contrat et elle n'opposait l'État de Grenade qu'à son contractant direct, c'est-à-dire la société RSM Production Corporation. ${ }^{75}$ L'ensemble des demandes ayant été rejeté, une nouvelle requête arbitrale a été introduite devant le CIRDI, mais cette fois-ci sur le fondement du TBI conclu entre l'État de Grenade et les États-Unis. Par ailleurs, outre la société RSM Production Corporation, trois actionnaires de celle-ci étaient dans cette seconde procédure également demandeurs.

Une grande partie des débats qui se sont déroulés au cours de cette seconde procédure visaient donc à déterminer la portée sur celle-ci de l'effet de collateral estoppel, c'est-à-dire de l'autorité positive de chose jugée, de la première sentence. Or, le tribunal arbitral a posé un certain nombre de jalons quant à la mise en œuvre de celle-ci dans les procédures CIRDI. Tout d'abord, la sentence admet que cet effet positif puisse aller au-delà

question, v. not. W. Ben Hamida, «L’arbitrage État-investisseur face à un désordre procédural: la concurrence des procédures et les conflits de juridictions», op. cit., p. 569.

73 Rachel S. Grynberg, Stephen M. Grynberg, Miriam Z. Grynberg, and RSM Production Corporation v. Grenada, aff. $\mathrm{n}^{\circ} \mathrm{ARB} / 10 / 6$, S. du 30 novembre 2010, \ 7.1.2. On notera que, pour procéder à cette affirmation, le tribunal arbitral se fonde essentiellement sur un arrêt de la Cour suprême des États-Unis d'Amérique (Southern Pacific Railroad Co v. United States, 168 U.S.1, 48-49 [1897]), ibid., \$ 7.1.3.

74 Ibid., \ 7.1.2. On notera que pour procéder à cette affirmation, le tribunal arbitral se fonde essentiellement sur un arrêt de la Cour suprême des États-Unis d'Amérique (Southern Pacific Railroad Co v United States, 168 U.S.1, 48-49 [1897]), ibid., S 7.1.3.

75 RSM Production Corporation v. Grenada, aff. n ARB/05/14, S. du 13 mars 2009. Une procédure d'annulation devant un comité ad hoc est pendante dans cette affaire. 
des seules parties à la première procédure, c'est-à-dire plus exactement qu'il puisse s'étendre aux actionnaires de la société investisseur direct. ${ }^{76}$ Ensuite, un fondement juridique à l'autorité positive de chose jugée est identifié par le tribunal arbitral à travers l'article 53-1 de la Convention de Washington, aux termes duquel «la sentence est obligatoire à l'égard des parties et ne peut être l'objet d'aucun appel ou autre recours, à l'exception de ceux prévus à la présente Convention ». ${ }^{77}$

En reconnaissant et en mettant en œuvre le principe de l'effet positif de chose jugée à travers la notion de collateral estoppel, et en lui assignant un fondement dans le système du CIRDI, Rachel S. Grynberg, Stephen M. Grynberg, Miriam Z. Grynberg, and RSM Production Corporation and others v. Grenada illustre parfaitement ce syncrétisme des traditions juridiques que le droit des investissements internationaux et sa pratique arbitrale sont en train de bâtir.

\section{References}

\section{Livres}

Daillier, P., Forteau, M. et Pellet, A., Droit international public, 8éd., LGDJ, Paris, 2002.

Dolzer, R. \& Schreuer, Ch., Principles of International Investment Law, Oxford University Press, Oxford, 2008.

Wälde, Th. (éd.), Energy Charter Treaty: An East-West Gateway for Investment and Trade, Kluwer Law International, 1996.

\section{Articles}

Audit, M., «Droit des investissements internationaux. Contentieux arbitral international. Droit commun », JCL. Droit international, fasc. 572-65.

Ben Hamida, W., «Clause de la nation la plus favorisée et mécanismes de règlement des différends: que dit l'histoire ?», JDI, 2007.

Ben Hamida, W., "Investment Arbitration and Human Rights", TDM, 2007, 4, (5).

76 Rachel S. Grynberg, Stephen M. Grynberg, Miriam Z. Grynberg, and RSM Production Corporation and others v. Grenada, aff. n ARB/10/6, S. préc. du 30 novembre 2010, \ 7.1.7.

77 Ibid., \ 7.1.9. 
Ben Hamida, W., «L'arbitrage État-investisseur face à un désordre procédural: la concurrence des procédures et les conflits de juridictions», AFDI, 2005, pp. 564-602.

Ben Hamida, W., «La consolidation des procédures arbitrales », Gaz. Pal., 2006, 13-14.

Crawford, J., "Treaty and contract in investment arbitration", Arb. Int., 2008.

Crivellaro, A., "Consolidation of Arbitral and Court Proceedings in Investment Disputes", The Law and Practice of International Courts and Tribunals, 2005.

Cuniberti, G., "Parallel Litigation and Foreign Investment Dispute Settlement", ICSID Rev., 2006, pp. 381-426.

Dodge, W. S., "National courts and international arbitration: exhaustion of remedies and res judicata under chapter eleven of NAFTA", Hastings Int. and Comp. L. Rev., 2000.

Fadlallah, I., «La distinction Treaty claims - Contract claims et la compétence du CIRDI: faisons-nous fausse route ?», in Leben, Ch. (dir.), Le contentieux arbitral transnational relatif à l'investissement, LGDJ/Anthemis, Paris, 2006, pp. 205-216.

Gaillard, E., "Establishing jurisdiction through a most-favored-nationclause", New York Law Journal, 2005.

Gallagher, N., "Parallel proceedings: res judicata and lis pendens in contemporary problems", in Lew \& Mistelis (ed.), International arbitration, Kluwer Law International, 2006, pp. 329-356.

Hanotiau, B., "NAFTA consolidation decision under art. 1126 of the NAFTA", Transnational Dispute Management, 2005, 2, (5).

Kaufmann-Kohler, G., Boisson de Chazournes, L., Bonnin, V. \& Mbengue, M. M., "Consolidation of Proceedings in Investment Arbitration: How Can Multiple Proceedings Arising from the Same or Related Situations be Handled Efficiently ?’, ICSID Rev., 2006, pp. 59-125.

Lemaire, S., «Treaty claims et Contract claims: la compétence du CIRDI à l'épreuve de la dualité de l'État», Rev. arb., 2006, pp. 353-400.

Loncle, J.-P., «L'option d'arbitrage des traités de protection des investissements: Treaty claims vs. Contract claims», RD aff. int., 2005, pp. 3-12.

Mayer, P., «Contract claims et clauses juridictionnelles des traités relatifs à la protection des investissements », JDI, 2009, pp. 71-96.

Mayer, P., «Litispendance, connexité et chose jugée dans l'arbitrage international», in Liber Amicorum Claude Reymond. Autour de l'arbitrage, LexisNexis Litec, Paris, 2004. 
Santulli, C., Droit du contentieux international, Domat droit public, Montchrestien, Paris, 2005.

Shany, Y., "Jurisdictional competition between national and international courts: could international jurisdiction-regulating rules apply?”, Netherlands Yearb. Int. L., 2006, pp. 3-56.

Sperduti, G., «Des actions judiciaires intentées dans un État du chef de nationalisations et d'expropriations opérées dans un autre État», in Mélanges Ch. Rousseau, Pedone, Paris, 1974, pp. 249-266.

Stern, B., «Trois arbitrages, un même problème, trois solutions: les nationalisations pétrolières libyennes devant l'arbitrage international», Rev. arb., 1980, pp. 3-43.

Yannaca-Small, K., "Parallel Proceedings", in Muchlinski, P., Ortino, F. \& Schreuer, Ch. (ed.), The Oxford Handbook of International Investment Law, Oxford University Press, Oxford, 2008, pp. 1009-1048.

\section{Jurisprudence}

\section{a) Jurisprudence arbitrale}

AEs Corp. c. Argentine, aff. n ARB/02/17, SC du 26 avr. 2005.

Azurix Corp. c. Argentine, aff. n ARB/01/12, SC du 8 déc. 2003, JDI 2004.

Bayindir Insaat Turizm Ticaret Ve Sanayi A.S. c. Pakistan, aff. $\mathrm{n}^{\circ}$ ARB/03/29, SC du 14 nov. 2005.

Benvenuti et Bonfant c. Congo, aff. $\mathrm{n}^{\circ} \mathrm{ARB} / 77 / 2$, S. du 8 août 1980, S1.14, ICSID Rep. 1993.

BP c. Lybie, S. 10 oct. 1973, Rev. arb. 1980, p. 117.

Československa obchodní banka (CSOB) c. Slovaquie, aff. $n^{\circ}$ ARB/97/4, ICSID Rev. 1999.

CMS Gas Transmission Company c. Argentine, aff. $\mathrm{n}^{\circ} \mathrm{ARB} / 01 / 8, \mathrm{SC}$ du 13 juill. 2003, ICSID Rep. 2003, p. 492, JDI 2004.

Compañia de Aguas del Aconquija and Vivendi Universal S.A. c. Argentina, aff. n ARB/97/3, DCAH du 3 juill. 2002, ICSID Rev. 2004, JDI 2003.

Emilio Agustín Maffezini c. Espagne, aff. n ARB/97/7, SC du 25 janv. 2000, ICSID Rev. 2001.

Enron Corporation and Ponderosa Assets c. Argentine, aff. n ${ }^{\circ}$ ARB/01/3, SC du 14 janv. 2004.

Eureko B.V.c. Rép. de Slovaquie, CPA n 2008-13, S. du 26 oct. 2010, Rev. arb. 2011. 
Gabon c. Sté Serete S.A., aff. $\mathrm{n}^{\circ} \mathrm{ARB} / 76 / 1$.

Gouvernement de la Province du Kalmantan de l'Est c. PT Kalim Prima Coal, aff. $\mathrm{n}^{\circ} \mathrm{ARB} / 07 / 3$.

Hulley Enterprises Limited (Cyprus) c. Russie, Yukos Universal Limited (Isle of Man) c. Russie et Veteran Petroleum Limited (Cyprus) c. Russie, CPA n ${ }^{\circ} \mathrm{AA}$ 226, AA 227 et AA 228, 3 SC du 30 novembre 2009.

Lanco International, Inc. c. République Argentine, aff. n ARB/97/6, SC du 8 décembre 1998, ILM 2001, vol. 40, pp. 457-473.

Lauder c. Rép. tchèque, S. ad hoc du 3 sept. 2001.

Liamco c. Lybie, S. du 12 avr. 1977, Rev. arb. 1980.

Noble Energy Inc. and Machala Power Cia. Ltd. c. Équateur et Consejo Nacional de Electricidad, aff. $\mathrm{n}^{\circ} \mathrm{ARB} / 05 / 12$, SC. du 5 mars 2008.

Occidental Exploration and Production company c. Ecuator, S. LCIA du 1er juill. 2004.

Occidental Petroleum Corp. and Occidental Exploration and Production Co. c. Équateur, aff. $\mathrm{n}^{\circ} \mathrm{ARB} / 06 / 11, \mathrm{~S} . \mathrm{du} 9$ sept. 2008.

Plama Consortium Ltd. c. Bulgarie, aff. n ARB/03/24, SC du 8 févr. 2005, \223, ICSID Rev. 2005.

Rachel S. Grynberg, Stephen M. Grynberg, Miriam Z. Grynberg, and RSM Production Corporation v. Grenada, aff. n ARB/10/6, S. du 30 novembre 2010.

Saipem S.p.A. c. Bangladesh, aff. $\mathrm{n}^{\circ}$ ARB/05/7, SC du 21 mars 2007, ICSID Rev. 2007.

Salini Construttori S.p.A. et Italstrade S.p.A. c. Royaume du Maroc, aff. $\mathrm{n}^{\circ}$ ARB/00/4, SC du 23 juillet 2001, JDI 2002, pp. 196-209.

SGS c. Pakistan, aff. n ARB/01/13, SC. du 27 août 2003, 』182, JDI 2004.

Siemens A.G. c. Argentine, aff. $\mathrm{n}^{\circ} \mathrm{ARB} / 02 / 8, \mathrm{SC}$ du 3 août 2004, \180, JDI 2005.

Tanzania Electric Supply Company Limited c. Independent Power Tanzania Ltd., aff. $\mathrm{n}^{\circ} \mathrm{ARB} / 98 / 8, \mathrm{~S}$. du 12 juillet 2001.

Telenor Mobile Communications AS c. Hongrie, aff. $\mathrm{n}^{\circ} \mathrm{ARB} / 04 / 15, \mathrm{~S}$ du 13 sept. 2006, ICSID Rev. 2006, JDI 2007.

Texaco Calasiatic c. Lybie, S. du 17 janv. 1977, AFDI 1977.

Waste Management, Inc. c. Mexique ( $\left.{ }^{\circ} 2\right), \mathrm{ARB}(\mathrm{AF}) / 00 / 3$, Mexico's Preliminary Objection concerning the Previous Proceedings, décision du Tribunal du 26 juin 2002, ICSID Rep. 2004.

Waste Management, Inc. v. United Mexican States, ARB(AF)/98/2, DC du 2 juin 2000, ILM 2001, vol. 40, ICSID Rev. 2000. 


\section{b) Jurisprudence étatique}

\section{i. Canada}

Federal Court of Canada, 13 janv. 2004, Attorney General of Canada v. S.D. Myers, 8 ICSID Rep. 194.

Ontario Superior Court of Justice, 3 déc. 2003, United Mexican States $v$. Feldman Karpa, 8 ICSID Rep. 500.

Supreme Court of British Columbia, 2 mai 2001, United Mexican States $v$. Metalclad Co, 5 ICSID Rep. 236.

ii. Etats-Unis

Banco National de Cuba v. Sabbatino, 376 U.S. 398 (1964).

U.S. District Court for the District of Columbia, 31 oct. 2005, 10 IC.SID Rep. 448

iii. France

CA Paris, 25 sept. 2008, Rép. tchèque c. Pren Nreka, D. 2009; RD aff. int. 2009; Gaz. Pal. 14-16 déc. 2008; RGDI publ. 2008.

iv. Grande-Bretagne

High Court of Justice, 29 avr. 2005, Republic of Ecuador v. Occidental Exploration and Production Co. [2005] EWCA Civ. 1116; [2006] QB 432 (CA).

v. Namibie

High Court (Namibie), 6 mars 2008, Kessl et al. c. Minister of Lands and Resettlement, RD aff. int. 2008.

vi. Suède

Czech Republic c. CME Czech Republic, aff. T8735-01, 9 ICSID Rep. 439.

vii. Suisse

T. fédéral, 10 nov. 2005, France Telecom c. Liban, aff. 4P.98/2005/svc.

T. fédéral, 7 sept. 2006, Saluka c. Tchéquie, aff. 4P.114/2006/bie. 